\title{
Oranas
INVERSIÓN DEL RECURSO-TIEMPO EN LA MONITORIZACIÓN DE EMBARAZADAS DE BAJO RIESGO POR EL EQUIPO DE SALUD DE LA FAMILIA ${ }^{1}$

Maeda ST, Ciosak SI. Inversión del recurso-tiempo en la monitorización de embarazadas de bajo riesgo por el equipo de salud de la familia. Rev Latino-am Enfermagem 2005 março-abril; 13(2):188-94.

Se trata de un estudio sobre gestantes de bajo riesgo en la unidad de salud de la familia. Tuvo como objetivos describir el recurso tiempo invertido por los equipos y analizar su configuración en el proceso del control prenatal, en la población seleccionada. Se basó en datos de historias clínicas de 47 mujeres, con edades comprendidas entre 20 a 29 años, residentes en el Municipio de Sao Paulo, abarcando períodos del 2001 al 2003. Las gestantes fueron clasificadas según los cuidados de salud desarrollados durante la fase del embarazo: sin problemas de salud, con intervención temprana de especialistas y aquellas con tratamiento odontológico. En los tres grupos, se verificó la inversión de mayor tiempo en las consultas realizadas por los profesionales de nivel superior y en las visitas en los domicilios, por los agentes comunitarios. La mediana del tiempo global, por gestante, fue de $10 \mathrm{~h}$ para el primer grupo, $12 \mathrm{~h}$ para el segundo y el tercero obtuvo una media de $12 \mathrm{~h}$. El índice del tiempo invertido en la atención directa varió de 59,40\% a 80,51\%.

DESCRIPTORES: atención prenatal; personal de salud; salud de la familia; factores de tiempo

\section{INVESTMENT OF TIME RESOURCE IN PRENATAL CARE BY FAMILY HEALTH STAFF}

This study is about low-risk pregnant women attended by a family health unit. It aims to describe the time resource the health staff invested and to analyze its configuration in the prenatal monitoring process. Data were collected from 47 women between 20 and 29 years old, who were attended from 2001 to 2003, in the eastside of São Paulo City. The pregnant women were classified according to the health care they received: a first group without health problems, a second with early expert intervention and a third with patients needing dental treatment. In the three groups, more time was invested by health professionals with higher education, followed by community health agents during home visits. Average total time per pregnant woman was 10 hours for the first group and 12 hours for the second and the third group. The time invested in direct care ranged from $59.40 \%$ to $80.51 \%$.

DESCRIPTORS: prenatal care; health personnel; family health; time factors

\section{INVESTIMENTO DO RECURSO-TEMPO NA MONITORIZAÇÃO DAS GESTANTES DE BAIXO RISCO PELA EQUIPE DE SAÚDE DA FAMÍLIA}

Trata-se de um estudo com gestantes de baixo risco, atendidas na unidade de saúde da família. Teve como objetivos descrever o recurso-tempo investido pela equipe de saúde e analisar a sua configuração em equipe, no processo de monitoramento pré-natal. Os dados foram baseados nas histórias clínicas de 47 mulheres, na faixa etária de 20 a 29 anos, atendidas entre 2001 e 2003, na zona leste do município de São Paulo. As gestantes foram classificadas em três grupos: sem problemas de saúde, com tratamento odontológico e com intervenção pontual de especialistas. Evidenciou-se maior tempo investido por profissionais de nível universitário, seguido de agentes comunitários. A mediana do tempo global, por gestante, foi de $10 \mathrm{~h}$ para o primeiro grupo, $12 \mathrm{~h}$ para o segundo e o terceiro obteve média de $12 \mathrm{~h}$. 0 índice do tempo apurado para 0 atendimento direto variou de $59,40 \%$ a $80,51 \%$.

DESCRITORES: cuidado pré-natal; pessoal de saúde; saúde da família; fatores de tempo

\footnotetext{
${ }^{1}$ Parte del proyecto de invetigación Dimensión de Costos: atención prenatal en el Programa de Salud de la Familia, de Sayuri Tanaka Maeda; ${ }^{2}$ Enfermera, Economista, Profesor Doctor, e-mail: sayuri@usp.br; ${ }^{3}$ Enfermera, Profesor Doctor, Director del Departamento de Enfermería Del Real, Benemérita Sociedade Portuguesa de Beneficência, e-mail: siciosak@usp.br. Escuela de Enfermería de la Universidad de São Paulo
} 


\section{INTRODUCCIÓN}

$\boldsymbol{E}$ | programa de atención a la mujer ha sido una de las prioridades en las políticas de la salud pública, dada la magnitud e importancia histórica de la mujer en la sociedad en las dos últimas décadas del siglo XX, tanto con relación a su contribución social, como en el ámbito político y económico.

Entre los cinco subprogramas del Programa de Atención Integral a la Salud de la Mujer (PAISM), implementado desde 1987 en Sao Paulo, la atención prenatal es de permanente inversión, en el primer nivel de la jerarquía de asistencia. El éxito de la cobertura prenatal constituye uno de los desafíos del Sistema Único de Salud (SUS), por ser amplio reflejo del grado de compromiso de los profesionales, por la elevada calidad de la asistencia, así como la mejoría de la capacidad organizacional de los servicios de salud.

Un estudio concerniente a la gestión de referencia y contrarreferencia, centralizado en la atención del ciclo obstétrico, reveló la existencia de problemas estructurales en unidades de atención básica, consultorios externos de especialidad y atención hospitalaria. Se constató una baja adhesión de las mujeres al control de la evolución del proceso de gestación. Fue verificada simultáneamente una falla en la estrategia de los servicios ofrecidos a estas embarazadas. En consecuencia, ocurrieron disfunciones institucionales provocando desarticulaciones entre los niveles de asistencia, hechos importantes que generaron la reducción de la capacidad de las instituciones para responder técnica y socialmente a las necesidades de las mujeres, con una inevitable pérdida de la unicidad del sistema de salud ${ }^{(1)}$.

Sobre este particular, se identificó la necesidad de desarrollar una tecnología apropiada en la estructura de la atención básica, para los gerentes, tomando en consideración la mejoría del acceso, accesibilidad y el aspecto integral de la asistencia al sistema del distrito de salud ${ }^{(1)}$.

Por lo tanto, estos resultados se refieren a otro enfoque sobre la gerencia de las unidades y los esfuerzos desarrollados en la consecución de programas, pues los gerentes no conseguían evaluar la relación costo-beneficio, visto que los recursos eran distribuidos de forma inespecífica, es decir, sin una noción real de la inversión para cada programa implementado.

El Programa de Salud de la Familia (PSF) se encuentra en expansión en el SUS con la intención de reorganizar el modelo asistencial con enfoque en la promoción de la salud y prevención de la enfermedad en el ámbito familiar.

Uno de los presupuestos filosóficos más discutidos, con gran potencial de transformación del modelo asistencial, es el trabajo en equipo descrito en el PSF conforme la NOB/96 ${ }^{(2)}$.

En la vigencia del SUS y en la búsqueda de implementación de los modelos de atención, existe un énfasis en tecnologías que enfocan la humanización en la atención, ejemplo de propuestas de formación y establecimiento de vínculo ${ }^{(3)}$ y el proceso de "acogimiento"(3). Siendo así se integraron nuevos patrones de relación profesional en la prestación de servicios, requiriendo de los profesionales adecuación del tiempo a ser invertido en esa producción.

La práctica de trabajo en equipo fue introducida en los procesos de producción industrial, con intención de renovar sistemas gerenciales tanto en el ámbito del mercado competitivo, como en las instituciones gubernamentales.

Se cree que la propuesta de proyecto colectivo expresa diferentes formas de creación de los profesionales ${ }^{(4)}$, por consiguiente, habrá posibilidad de retratar cómo influye la utilización del tiempo entre los miembros del equipo y también cómo se da a través de la participación de las usuarias en el proceso de atención.

Para tal discusión, en este trabajo, fue utilizado el aporte de las autoras que teorizan el trabajo en equipo en el PSF, como una relación de proceso y en permanente articulación ${ }^{(5)}$.

El arte realizado a través del trabajo y el tiempo utilizado reproducen, en parte, la importancia que se da a los momentos de producir respuestas en el proceso de atención de la dimensión individual, de grupo, de familias y del colectivo. Partiendo del presupuesto que los profesionales asumen la inversión del tiempo en equipo, surgió la siguiente inquietud - ¿Qué configuración de tiempo se da en esa modalidad de trabajo? Conforme estas consideraciones se delimitaron recurso-tempo* invertido** foco central de discusión del costo del personal.

El tiempo no es elástico, o sea, no podemos guardarlo 0 estirarlo, mientras sea visto como tiempo físico controlado por el reloj. Este modifica el sentido, según sus valores culturales en un contexto vivido por las personas o grupos. En la lógica empresarial, concerniente al trabajo, el valor tiempo es inmediatamente proporcional a la productividad e inversamente proporcional al consumo, otorgándole un valor económico-financiero.

El recurso-tiempo, así interpretado, como tiempo cronológico, se remite al concepto utilitarista del tiempo como un elemento productor de riqueza, reproduciendo los valores subyacentes a las instituciones que tienen como objetivo la eficiencia económica en razón de la supervivencia de las empresas en el mercado ${ }^{(6-7)}$. Así, el recursotiempo se tornó un mediador esencial y condicionante, interdependiente en la construcción de significados en la producción de servicios.

Frente a tal reconocimiento, la propuesta de trabajo en equipo

\footnotetext{
* recurso-tiempo: expresión creada por los autores para delimitar el significado del tiempo como uno de los recursos esenciales en el desarrollo de la asistencia, así como en la gerencia y producción de servicios

** invertido: término proveniente del campo económico, asumido por los autores con la intención de expresar la expectativa de retorno o respuesta de las acciones
} 
hace relativos los mecanismos de subordinación y control, abriendo espacio para el ejercicio de la autonomía en la producción de los servicios de salud. Según este raciocinio se proyectó el tiempo como un recurso, aunque abstracto, en la dimensión individual y colectiva.

Al incluir los procesos de trabajo en equipo, vínculo y acogimiento como condicionantes de los cambios en las operaciones de salud, se vislumbra mudanza del tiempo equilibrado, del eje de atención guiado por la racionalidad cronológica, al mismo tiempo que se reitera la producción de la subjetividad humana. Es de fundamental importancia comprender que el tiempo es una fuerza psicológica, fundamental en nuestras vidas, una fuente de poder que debe ser mediada para ayudar a pensar de forma mas efectiva y ayuda a comprender las emociones con claridad y tomar decisiones con más confianza ${ }^{(6)}$.

Frente a lo expuesto, las autoras de este trabajo propusieron como objetivos específicos describir el recurso-tiempo invertido en la monitorización de las embarazadas de bajo riesgo y analizar cómo se conforma en la dinámica de los equipos en ese proceso de atención.

\section{METODOLOGÍA}

La necesidad de conocer el tiempo surgió al investigar gastos con el personal, teniendo que identificar la parcela de la remuneración de los profesionales, en función de la monitorización de las embarazadas realizando un recorte de los instantes del conjunto tiempo.

Se tomó como objeto de estudio el recurso-tiempo invertido por los equipos de salud, valorizando sus procesos de producción relacionados al prenatal, en un Programa de Salud de la FamiliaQUALIS, en el período de agosto del 2001 a mayo del 2003.

El Programa está localizado en el Distrito de Sapopemba, región sur este de Sao Paulo, administrada por la Fundación Zerbini, organización que trabaja en sociedad con la Secretaria de Salud del Estado de Sao Paulo desde 1998. Responde por un área de alcance definida, dividida en cuatro macro áreas y éstas en 22 micro áreas, abrigando 4.700 familias. En total existen en las macro áreas aproximadamente 1.000 familias y en las micro áreas cerca de 200 familias.

La gerencia de la unidad cuenta con un Director, un Consejo Gestor y 52 trabajadores que son copartícipes de la gestión.

La estructura básica del trabajo de esa unidad esta compuesta por cuatro equipos y cada cual responde por una macro área. Los equipos están compuestos por un médico de familia, una enfermera de familia, dos auxiliares de enfermería y cinco a seis agentes comunitarios de salud, conforme la extensión del número de familias. Integra también un equipo de odontología que actúa con tres cirujanos dentistas, un técnico de higiene bucal y tres auxiliares de salud bucal, que atendían por demanda espontánea.
En el período establecido para el estudio, con intención de facilitar la recolección de datos, fue escogido un grupo homogéneo de embarazadas que posibilitó un análisis más fidedigno del objetivo propuesto.

Como fuentes de consultafueron utilizados los libros de registro de las agentes comunitarias referente al censo de embarazadas por micro áreas, siendo ambos actualizados a cada mes y también fueron utilizadas las historias clínicas familiares. La historia clínica constaba de cuatro números en secuencia, identificando las macro áreas, micro áreas, las usuarias y sus familias. De esta forma, la ficha clínica de las embarazadas fue localizada por el número de registro individual, entre otros miembros familiares. Fueron consultados también los datos del Sistema de Información de Atención Básica (SIAB) para complementar informaciones relacionadas con la familia.

Para la definición de la muestra, se identificaron inicialmente 151 embarazadas censadas en 2002. En el período establecido para la investigación 24 mujeres atendían al criterio establecido, 44 fueron excluidas por edad y 83, la mayoría, por motivo de transferencia a los consultorios externos de especialidades.

Frente a la reducida muestra, decidimos ampliar el período. De esta forma fueron incluidas 17 embarazadas a mediados del 2002 y que dieron a luz hasta junio del 2003 y seis mujeres más embarazadas en el segundo semestre del 2001 y que dieron a luz en el 2002. Por lo tanto, fueron seleccionadas 47 mujeres, clasificadas como embarazadas de bajo riesgo, en el grupo de edades de 20 a 29 años, y con acceso mínimo a cuatro consultas, en el calendario de atención prenatal.

La delimitación del grupo según edades fue orientada por la clasificación en la literatura, en: hasta 19 años, de 20 a 29 años y de 30 años a más. El de 20 a 29 años es considerado el mejor grupo de edades en el desarrollo del embarazo y parto, por la condición biológica y madurez de la mujer ${ }^{(8)}$. A pesar que algunos estudios presentan a las mujeres de 18 a 24 años como el mejor grupo señalan que de exceder esos límites existe la probabilidad de incidencias de distocias ${ }^{(9)}$.

El instrumento elaborado para la recolección de datos de las embarazadas, constó de datos generales y los procedimientos de rutina para la monitorización del embarazo.

El proyecto fue evaluado y aprobado por el Comité de Ética en investigación de la Coordinación de Desarrollo de la Gestión Descentralizada-COGest, de la Secretaria Municipal de Salud y su seguimiento fue a través de informes periódicos.

A continuación, fue solicitada la autorización para realización de esta investigación a la dirección de la Fundación Zerbini por medio de una carta explicando el propósito y los objetivos deseados, así como también la conducción del pre test para validación del instrumento.

Para el análisis de los datos se utilizó el Programa Access cuyo banco de datos pasó por fases de test, permitiendo la introducción de ajustes necesarios y visualizar la trayectoria de cada embarazada. 
Las tablas extraídas fueron analizadas en el Programa Excel y distribuidas por tipo de cuidados previamente definidos por grupo: sin problemas de salud, con tratamiento odontológico y con intervención de especialistas, para facilitar la descripción y el análisis.

La aprehensión del tiempo de ese proceso de prestación de la asistencia fue posible mediante una proyección de pasos planeados durante la monitorización del embarazo de bajo riesgo, instituido por los equipos, compartido por la embarazada y su familia. Además de tomarse como referencia los protocolos internos y las normas del Ministerio Salud ${ }^{(10)}$.

Los momentos de interacción de las embarazadas con los equipos de la unidad fueron apropiados considerando que la sucesión de hechos no es lineal. Así, los pasos son reflejos de las variaciones de atención por embarazada, desde la recepción, las consultas, las faltas, los exámenes y problemas. Con seguridad representan situaciones encadenadas con duración limitada, que no se repiten para los profesionales y usuaria, constituyendo el tiempo una oportunidad en relación con los propósitos de cada acción.

La unidad de medida del tiempo fue expresada en minutos, puesto que para algunos procedimientos se invierten menos de 30 minutos. La sumatoria de estos minutos fueron convertidos en horas.

Para la apropiación del tiempo invertido por los profesionales en la asistencia prenatal, se respetó un tiempo patrón destinado a las consultas en el ámbito de la administración QUALIS/PSF, por ejemplo, el tiempo de consultas individuales está estimado en 30min.

Las demás actividades, relacionadas al tiempo, como grupos, aplicación de vacunas, recolección de materiales, visita y atención domiciliaria, incluyendo al personal administrativo, fueron estimadas mediante observaciones empíricas de las prácticas y la experiencia de las investigadoras.

Referente al tiempo invertido en la atención odontológica, se evaluó el tratamiento de cada consulta y fue atribuido el tiempo por participación de los profesionales.

\section{RESULTADOS Y DISCUSIÓN}

En el período propuesto, fueron monitorizadas las 47 embarazadas que cumplían con los criterios de inclusión y clasificadas en tres subgrupos: 37 embarazadas $(78,72 \%)$ presentaron una evolución sin problemas de salud*; 8 (17,03\%) fueron sometidas a la asistencia odontológica, además de la monitorización, y 2 (4,25\%) tuvieron intervención puntual de especialidades.

El análisis tuvo en consideración esas particularidades de los grupos, siendo que el enfoque más profundo se centralizó en las 37 embarazadas sin problemas de salud por representar un mayor contingente en la población estudiada. Las medidas, mediana y media, de tendencia central fueron adoptadas para evaluar el tiempo invertido en el proceso de atención.

Tabla 1 - Recurso - tiempo invertido en la atención de las embarazadas sin problemas de salud por los equipos de las macroáreas y administrativa. Programa de Salud de la Familia-QUALIS en minutos totales. Distrito de Sapopemba. São Paulo, 2003

\begin{tabular}{lcccc}
\hline & $\mathbf{H}_{\text {equipomacroárea }}$ & $\mathbf{H}_{\text {admin }}$ & \multicolumn{2}{c}{ Totales } \\
\hline Unidad & min. & $\min$ & $\min$ & horas \\
Tiempo invertido & 16.590 & 5.805 & 22.395 & 373,26 \\
Mediana & 440 & 155 & 595 & 9,92 \\
Media & 448,37 & 156,9 & 605,27 & 10,09 \\
\hline
\end{tabular}

El tiempo invertido en la monitorización (Tabla 1), embarazadas sin problemas de salud, resultó en la mediana 440min y media 448,37min

Cabe destacar que en la Tabla 1 y en las demás Tablas, el desdoblamiento de la Hequipomacroárea comprendió la realización de la prueba de embarazo (Hatdiag), consultas de la enfermera (Hcenferm ), consulta con el médico general (Hcmedgener), trabajo de supervisión de la enfermera en la recolección de materiales para exámenes (Hexlabenf), ejecución de la recolección de materiales por el auxiliar de enfermería (Hexlabauxe), atención en grupo (Hatgrupo), aplicación de las vacunas (Hvacuna), reuniones del equipo (Hadequipo) y el trabajo del agente comunitario (Hagcom), exceptuando los momentos de los trabajos administrativos.

Este conjunto de acciones dedicadas a 37 embarazadas fue agrupado en un intervalo de $2 h$ y más de $2 h$ y la mayoría permaneció en el grupo del tiempo invertido de 8 a $10 \mathrm{~h}$.

Al decomponer la Hequipomacroárea, fue percibido que el diferencial del recurso-tiempo oinvertido por los profesionales estaba relacionado al número de consultas médicas, de enfermería y visita domiciliaria realizadas, éstas últimas, por auxiliares de enfermería y agentes comunitarios.

Hubo una variación del número de consulta médica por embarazada. El grupo que abarcó a las 37 embarazadas, reveló una inversión de 8 a 10h, lo que correspondió de 1 a 7 consultas, dando una media de 2,5 por médico. En relación a la consulta de enfermería se observó una inversión de 2 a 10 consultas, lo que correspondió a una media de 5,1 consultas por enfermera. Es interesante observar que la media general de consulta, entre enfermeras y médicos, fue en torno de 7,5 por embarazada. Este resultado revela el nivel de cobertura en consultas individuales, más allá de lo esperado, pues la

\footnotetext{
* La denominación sin problemas de salud tuvo por objetivo establecer distinción entre os subgrupos seleccionados para el presente estudio. Pues, algunas gestantes, entre las 37, presentaron episodios de infección y demás situaciones provenientes del proceso de gestación
} 
recomendación ministerial para embarazos normales de bajo riesgo es en lo mínimo 6 consultas.

La captación precoz constituye un aspecto positivo pues fue verificado, en el registro, que el uso de recursos de diagnósticos del embarazo es una de las primeras medidas del servicio para conseguir la adhesión de las embarazadas. Constatado el embarazo, por la enfermera, se marcaba la próxima consulta y simultáneamente los agentes comunitarios eran informados sobre la necesidad programar el respectivo censo de la embarazada.

Los exámenes de rutina así como los resultados también fueron constatados en los registros de solicitación. La atribución de tiempo para cada examen fue estandarizado, a pesar que cada gestante tenía historia clínica diferente en relación a la frecuencia de solicitudes de exámenes de diferente índole. El tiempo de supervisión de la enfermera fue estimado en 5 minutos invertido en el apoyo brindado al personal en esas actividades. Fueron adicionados 10 minutos del tiempo a los auxiliares de enfermería que actuaban en parejas durante la recolección de materiales para exámenes.

La realización de visitas domiciliarias de los médicos y de las enfermeras fue ejecutada en casos puntuales de intervención a las embarazadas y sus familias, motivadas por crisis de hipertensión arterial, necesidad de supervisión próxima al parto y otras.

Respecto a las vacunas, se observó una asistencia regular en casi la totalidad de la muestra, habiendo apenas 3 embarazadas que fueron orientadas a la aplicación de la dosis de refuerzo de la vacuna dupla adulto (dT).

La atención en el domicilio, realizada por los auxiliares de enfermería, se justifica por la ejecución de procedimientos, seguimiento de las terapéuticas establecidas, diversas orientaciones a la familia de los postrados en camas o en reposo, y recolección de materiales para exámenes. Se verificó también que 6 embarazadas recibieron este apoyo, que influyó en la elevación del tiempo dedicado a ellas apuntando un tiempo variable entre 15 a 105 min.

En cuanto a la actuación de los agentes comunitarios en los domicilios, se estimó un tiempo patrón destinado a desarrollar censos, con la intención de actualizar las informaciones que subsidiaba las acciones de intercambio, como las actividades del equipo y la función de acoger quejas de las familias. En este sentido, el tiempo gastado es representativo, si comparado a las horas requeridas por las consultas individuales. De hecho, se estima que gran parte de la producción de agentes comunitarios ocurrió con familias y en la interlocución con los miembros del equipo.

El tiempo consumido en reuniones, también fue computado como actividad gerencial del equipo, tomando en cuenta la participación de todos los elementos. Se llegó a un acuerdo siendo un "pool" de tiempo, aproximadamente 10,0\% del total del tiempo del equipo, destinado a la discusión o ajustes en la monitorización de la situación de las embarazadas, frente a la reconocida prioridad de esa población, en el ámbito de la atención básica.

En cuanto a los servicios administrativos éstos fueron agrupados, para una mejor comprensión, en actividades de apoyo para la consecución de la monitorización de las embarazadas. Fue asumido un tiempo patrón, que los auxiliares administrativos invierten en la transacción de las historias clínicas, específicamente en consultas individuales (cita marcada u otras actividades eventuales), multiplicado por el número de consultas realizadas. La mediana encontrada fue de 20 min y el valor medio de 22 min en el período de gestación.

Fue agregado un tiempo de apoyo sobre la actividad de distribución de productos farmacéuticos, recetados por médicos y enfermeras, gastando 4 minutos por embarazada. De este modo, fue establecido un patrón de 15min de consumo de tiempo, para esa tarea, durante el embarazo.

La hora gerente (Hger) comprende la participación en todas las reuniones de equipos, mantenimiento de la infra-estructura de abastecimiento de materiales y medicamentos, recursos humanos, implementaciones de acciones, supervisión de personal administrativo y producción de informaciones relativas a las embarazadas.

La Tabla 2 se refiere a 8 embarazadas que se sometieron al tratamiento odontológico, además de monitorización de la evolución del embarazo.

Comparando estos resultados con los de la Tabla 1, relativos a los equipos de la macro área, encontramos una variación negativa de la mediana -16\% y media de -6,77\%. Esta disminución del tiempo, centrado en la monitorización del embarazo, ocurrió en virtud del tratamiento odontológico que veremos más adelante.

Tabla 2 - Tiempo invertido por el conjunto de los equipos en la monitorización del embarazo y el tratamiento odontológico en el Programa de Salud de la Familia. Distrito de Sapopemba. Sao Paulo, 2003

\begin{tabular}{cccccc}
\hline & $\mathbf{H}_{\text {odonto }}$ & $\mathbf{H}_{\text {equipomacroarea }}$ & $\mathbf{H}_{\text {admin }}$ & \multicolumn{2}{c}{ Total } \\
\hline Unidad & min. & min. & min. & min. & horas \\
Mediana & 109 & 378 & 162 & 724 & 12,06 \\
Media & 180 & 418 & 165 & 763 & 12,71 \\
\hline
\end{tabular}

Partiendo del presupuesto que las embarazadas mencionadas en la Tabla 2, deberían recibir cuidados semejantes a las de la Tabla 1, se evidenció una diferencia de horas invertidas por los equipos, en la columna de Htotales. En el tiempo de Htotales aumentadas comparado con el grupo sin problemas de salud, de la Tabla 1, los valores de la mediana de 12h 3' 6" y 12h 43' 6" indicaron una variación positiva de 21, 57\% y 25,96\%, por cuenta de la atención odontológica en relación con la Tabla 1.

Respecto al equipo de las macro áreas obtuvimos resultados de mediana que fue de $59,40 \%$ y media de $56,30 \%$ del total del tiempo 
invertido, habiendo una distribución homogénea del tiempo para cada embarazada. Sin embargo, en la composición general del tiempo, hubo una reducción porcentual de -10\% y -8,04\%, por cuenta de la atención odontológica (Tabla 2).

Una interpretación posible sería la dificultad en el distribución del tiempo por el acúmulo de la demanda de cuidados con relación al cumplimiento de la consulta marcada, sobrepuestas a las actividades domésticas. La Tabla 2 muestra que algunas embarazadas necesitaron mayor tiempo del equipo de odontología pues, conforme sus historias clínicas, frecuentaron innumeras secciones. Las demás se sometieron a intervenciones puntuales, como absceso, exodoncia y otras, indicando posible falla en cuidados preventivos.

Con relación al tiempo de la administración, las Tablas 1 y 2 , muestran mayor constancia de tiempo invertido, debido al criterio adoptado. Sin embargo, cuanto mayor demanda en la asistencia, mayor la correspondencia del tiempo para la administración.

Comparando la Tabla 1 y 3 , tomando como base la primera, la variación porcentual calculada fue positiva, indicando 4,51\% para mediana y media de 3,84\%.

Analizando la Tabla 3, verificamos que el diferencial fue debido al apoyo del equipo de salud mental, además de la monitorización prenatal.
Tabla 3 - Tiempo invertido por el conjunto de los equipos en la monitorización del embarazo e intervención puntual de los especialistas en el programa de embarazo. Distrito de Sapopemba. Sao Paulo 2003

\begin{tabular}{lcccccccc}
\hline & \multicolumn{2}{c}{$\mathbf{H}_{\text {equipomacroarea }}$} & \multicolumn{2}{c}{$\mathbf{H}_{\text {eqespecial }}$} & \multicolumn{2}{c}{$\mathbf{H}_{\text {admin }}$} & \multicolumn{2}{c}{ Total } \\
\hline Unidade & min & $\%$ & min & $\%$ & min & $\%$ & min. & horas \\
Media & 415 & 60,91 & 110 & 15,63 & 155 & 23,46 & 680 & 11,33 \\
\hline
\end{tabular}

El tiempo invertido por los especialistas, como médico psiquiatra, terapeuta ocupacional y psicólogo, además del equipo de macro áreas y de administración, tuvo una media de 11h19'48". El equipo, Hequipomacroárea marcó la presencia de 60,91\%, mientras que la Hequipoespecial resultó en 15,63\% del tiempo total (Tabla 3). El tiempo de las Hequipoespecial marcó una actuación puntual, conforme verificado en los registros clínicos y referencias de la enfermera, no caracterizando un seguimiento secuencial y continuo. Así mismo, la interrelación entre el equipo de salud mental y el equipo de la macro área aparentemente fluye, esto fue observado mediante registros de la evolución o la regresión del comportamiento de la usuaria, abordando sistemáticamente el contexto de las respectivas familias.

La variación porcentual de la media de Htotales, en vista del grupo sin problemas de salud, fue de 12,29\%.

Tabla 4 - Distribución del trabajo del equipo de la macro área, según tiempo invertido en la monitorización de las embarazadas. Distrito de Sapopemba. Sao Paulo, 2002

\begin{tabular}{|c|c|c|c|c|c|c|c|c|c|c|c|}
\hline & $\mathbf{H}_{\text {atdiag }}$ & $\mathbf{H}_{\text {cenferm }}$ & $\mathbf{H}_{\text {cmedgener }}$ & $\mathbf{H}_{\text {exlagenf }}$ & $\mathbf{H}_{\text {exlabauxe }}$ & $\mathbf{H}_{\text {atgrupo }}$ & $\mathbf{H}_{\text {adequipe }}$ & $\mathbf{H}_{\text {agcom }}$ & & Totale & \\
\hline Unidad & $\min$. & $\min$. & $\min$. & min. & min. & min. & $\min$. & $\min$. & min. & $\%$ & horas \\
\hline Mediana & 10 & 135 & 120 & 5 & 10 & 15 & 60 & 128 & 378 & 58,53 & 6,30 \\
\hline Media & 10 & 128 & 102 & 6 & 14 & 15 & 60 & 135 & 417 & 56,09 & 6,95 \\
\hline
\end{tabular}

La Tabla 5 ilustra los momentos de apoyo administrativo al proceso de atención a las embarazadas. Está compuesto por el movimiento dinámico de las historias clínicas (Hagadm), entrega de medicamentos (Hagadmmed) y el tiempo que la dirección participa en las reuniones del equipo y en la gerencia de las informaciones (Hger). Se observó mayor constancia de los tiempos por el criterio preestablecido para procedimientos administrativos. Se verificó que el tiempo dedicado por los agentes administrativos no fueron afectados con la variación de las necesidades de atención en la monitorización de ciclo de gestación y de la salud oral.

Retornando a las Tablas, el tiempo de la administración, destinado a la evolución de las embarazadas, se mostró idéntico a lo encontrado en las demás embarazadas, situándose entre 19,50\% a $27,43 \%$.

Lo que llamó la atención fue que, aunque busquen el mismo propósito de promover el embarazo saludable, inciden tiempos diferentes por embarazadas indicando que la atención está centralizada por necesidades identificadas. Así, el tiempo se torna elástico tanto para quien recibe los cuidados de salud como para quien programa la atención $^{(5,11-12)}$. Una de las cuestiones claves del cambio del modelo de atención debe residir en la conformación del tiempo invertido para desarrollar el trabajo.

Tabla 5 - Tiempo invertido por el Equipo de administración para la monitorización de las embarazadas. Programa de Salud de la Familia. Sapopemba. São Paulo 2003

\begin{tabular}{lccccccc}
\hline & $\mathbf{H}_{\text {agadm }}$ & $\mathbf{H}_{\text {agadmmed }}$ & $\mathbf{H}_{\text {ger }}$ & \multicolumn{3}{c}{ Subtotales } & Total \\
\hline Unidad & min. & min. & min. & min & horas & $\%$ & min. \\
Mediana & 30 & 12 & 120 & 162 & 2,70 & 21,57 & 724 \\
Media & 33 & 14 & 120 & 165 & 2,75 & 22,51 & 762 \\
\hline
\end{tabular}

Cabe resaltar el tiempo precioso invertido en el ejercicio de las profesiones de salud, pues el modo de atención permite hacer aflorar la valorización, oportunidad y dar forma al tiempo dedicado, siendo éste enfatizado en las necesidades de salud de las embarazadas y sus familias. Para que esta propuesta gane sustento en la práctica, es determinante que los profisionales percibran la diferencia entre la 
concepción del tiempo físico del reloj y el tiempo en la perspectiva de la cliente, a pesar que éste sea un aspecto subjetivo, proveniente de la sensibilidad humana ${ }^{(11-12)}$.

\section{CONSIDERACIONES FINALES}

Frente a los resultados con relación al tiempo invertido, en este Programa de Salud de la Familia, fue posible demostrar que el control prenatal, señaladas por el PAISM, fue un proceso viable para la mayoría de las embarazadas estudiadas. Dentro de esta realidad, el conjunto del tiempo encontrado, en mediana y media, demostró una práctica posible de ser ejecutada por el equipo. Se presupone que el principio de cooperación y articulación continua, entre servicio y familias, han hecho posible la obtención de resultados de adhesión al Programa de atención prenatal. En este contexto se verificó una gran participación de las enfermeras en el Programa.

La inversión del tiempo asumido por los equipos de las macro áreas, fue superior a 70,0\% en atención directa. Si adicionadas las horas asistenciales de especialistas y del equipo de odontología, puede observarse un 80,0\% para la monitorización.

Se concluye que todo esto es posible sólo con el compromiso de los equipos y la corresponsabilidad de las usuarias, resultante del vínculo y acogimiento que expresan la elasticidad del tiempo, en la atención prenatal ${ }^{(3)}$, a pesar que la esfera subjetiva de estos determinantes no aparezca como apropiadas en el tiempo. Se considera que el Programa monitorizado, basado en la necesidad de las usuarias, como núcleo de decisión, definirán el curso de las acciones sin perder de vista el compromiso con la organización y el nivel de productividad esperado por el equipo ${ }^{(4-6,10)}$.

\section{REFERENCIAS BIBLIOGRÁFICAS}

1. Maeda ST. Gestão de referência e contra-referência na atenção ao ciclo gravídico-puerperal: a realidade do Distrito do Butantã. [tese] São Paulo (SP): Escola de Enfermagem/USP; 2002.

2. Ministério da Saúde (BR). Norma Operacional Básica do Sistema Único de Saúde: NOB-SUS 01/96. Brasília: MS; 1997.

3. Merhy EE. Em busca da qualidade dos serviços de saúde e 0 modelo assistencial em defesa da vida. In: Cecílio LCO, organizador. Inventando a mudança na saúde. $2^{a}$ ed. São Paulo: Hucitec; 1997. p.117-60.

4. Shinyashiki GT, Trevizan MA, Mendes IAC. Sobre a criação e a gestão do conhecimento organizacional. Rev Latino-am Enfermagem 2003; 11(4):499-506.

5. Ciampone MH, Peduzzi M. Trabalho em equipe e trabalho em grupo no Programa de Saúde da Família. Rev Bras Enfermagem 2000; 53:143-7.

6. Bernhoeft R. Administração do tempo: um recurso para melhorar a qualidade de vida pessoal e profissional. São Paulo: Nobel; 1985.

7. Testa M. Análisis de instituciones hipercomplejas. In: Merhy EE, Onocko R, organizadores. Agir em saúde- um desafio para o público. São Paulo: Hucitec; 1997. p. 17-70.

8. Siqueira AAF, Tanaka ACD'LA, Santana RM, Almeida PAM. Mortalidade materna no Brasil, 1980. Rev Saúde Pública 1984; 18(6):448-65.

9. Rezende J. Anamnese e exame físico. In: Rezende J. Obstetrícia. $9^{a}$ ed. Rio de Janeiro. Guanabara Koogan; 2000. p.179-200.

10. Ministério da Saúde. Secretaria de Políticas de Saúde (BR). Assistência pré-natal: manual técnico. Brasília: MS; 2000.

11. Remen RN. O paciente como ser humano. São Paulo: Summus Editorial; 1993.

12. Doctors M, organizador. Tempo dos tempos. Rio de Janeiro: Zahar; 2003. 